(Aus dem Physiologischen Institut der Tierärztlichen Hochschule zu Dresden.)

\title{
Über den Magenmechanismus des Pferdes bei der Getränkaufnahme.
}

Studien zur vergleichenden Verdaungsphysiologie.

\author{
IV. Mitteilung. \\ Von \\ Arthur Scheumert.
}

(Mit 27 Textfiguren.)

Erst in neuerer Zeit hat man sich eingehender mit dem Verhalten der beim Trinken in den Magen gelangten Flüssigkeiten und ihrem Einfluss auf den Magenmechanismus befasst und dabei festgestellt, dass diese Flüssigkeiten sowohl den leeren als auch den gefüllten Magen in kurzer Zeit wenigstens zum grössten Teile verlassen. Eigentlich ergibt sich die Notwendigkeit grosser Entleerungen des gefüllten .Magens nach Flüssigkeitsaufnahme in vielen Fällen schon daraus, dass die aufgenommene Getränkmenge in einem Missverhältnis zu dem im Magen noch vorhandenen Raum steht. Am deutlichsten tritt dies bei den Einhufern hervor. Man denke z. B. an ein Pferd, das bei einer Magenkapazität von 10-15 Liter nach einer reichlichen Mahlzeit von $2 \mathrm{~kg}$ Hafer und $0,75 \mathrm{~kg} \mathrm{Heu}$, die nach dem Einspeicheln ungefähr ein Gewicht von $10 \mathrm{~kg}$ repräsentieren, 10, 12, ja 20 Liter Wasser trinkt, also mebr als der Magen überhaupt im leeren Zustande fassen kann. Dabei könnte natürlich auch ein Herausschwemmen des Mageninhaltes bewirkt werden. Es dürfte nicht schwer fallen, für einen zu seinen Mahlzeiten reichlich trinkenden Menschen das gleiche nachzuweisen.

Meines Wissens finden sich die ersten experimentellen Angaben über das Verhalten von Flüssigkeiten im Magen in der Physiologie von Gurlt ${ }^{x}$ ), der einen Versuch von Colemann anführt. Dieser ältere Autor liess ein Pferd viel

1) Gurlt, Lehrbuch der vergleich. Physiologie der Haussäugetiere, 2. Aufl., S. 141. Hirschwald, Berlin 1847. 
Wasser trinken und fand, dass dieses schon nach 6 Minuten durch den Pförtner und durch die dünnen Därme bis in den Blinddarm gelangt war. Gurlt selbst stellte auch Versuche an; er liess einem Pferd 8 Pfund Heu und 32 Pfund Wasser geben und es einige Minuten nach dem Trinken töten. Im Magen überhaupt fanden sich noch $31^{1 / 2}$ Pfund. Ein zweites Tier erhielt nach 12 stündigem Fasten 231/4 Pfund Wasser. Nachdem es "bald darauf" getötet worden war, fanden sich neben einigen Pfunden zerkauten Heues noch $\mathbf{7 1}^{1 / 4}$ Pfund Flüssigkeit im Magen. Einem dritten Pferde liess Gurlt 2 Stunden vor dem Tode 2 Pfund Heu und 4 Pfund Hafer reichen und ibm 301/4 Pfund Wasser zu trinken geben. Bei der Untersuchung fanden sich noch alle Nahrungsmittel, es fehlten aber 17 Pfund Wasser. Natürlich ist, wie auch Gurlt betont, die tatsächlich verschwundene Wassermenge eine viel grössere gewesen, da der beim Kauen verschluckte Speichel und der Magensaft nicht mit in Anrechnung gebracht worden sind. Bedenkt man, dass beim ersten Pferde auf die 8 Pfund Heu allein mindestens 32 Pfund Speichel und beim dritten Pferd auf die genossene Nahrung $4+16$, also 20 Pfund Speichel ergossen worden sind, so wird dies deutlich.

Später hat dann $\mathrm{Colin}^{1}$ ) ähnliche Versuche angestellt und ist zu gleichen Resultaten gelangt.

Darüber aber, in welcher Weise dieser rasche Durchtritt von Wasser durch den Magen erfolgt, hat lange Zeit Unklarheit geherrscht. Colin dürte hierüber bezüglich des Pferdes zum ersten Male auf Versuche gestützte Anschauungen geäussert baben. Er glaubt, dass in gewissen Fällen die Flüssigkeiten „sortent doucement par un courant dirigé du Cardia au Pylore sur la petite courbure", ohne Mageninhalt fortzuführen oder ihn zu verdünnen und seine Lagerung zu stören. In anderen Fällen hingegen sollen sich die Flüssigkeiten mit dem Mageninhalt völlig vermischen (ils en bouleversent tout les parties et les reduisent en bouillie) und ihn mit sich fortführen.

Auf dem 12. Kongress für innere Medizin 1893 berichtete dann v. Mering ${ }^{2}$ ) über seine bekannten Versuche an Hunden mit kurz hinter dem Pylorus angelegter Duodenalfistel. Er führte dabei aus, dass das aufgenommene Wasser den leeren Magen rasch und in relativ kurzer Zeit auch total verlässt, ohne dabei saure Reaktion anzunehmen. Moritz ${ }^{3}$ ) bestätigte in einer Diskussionsbemerkung diese Befunde und äusserte sich bezüglich des Verhaltens bei gefülltem Magen wie folgt: „Wenn wir sehen, dass Flüssigkeit den Magen so rasch verlässt, könnten wir zu der Ansicht kommen, dass es unzweckmässig sei, zum Essen auch zu trinken, da alsdann die Gefahr vorläge, dass die Speisen mit der Flüssigkeit vor gehöriger Einwirkung der Magenverdauung auf dieselben in den Darm geschwemmt würden. Nach meinen Beobachtungen ist indessen das Verhalten so, dass die Flüssigkeit nach einiger Zeit allein in den Darm

1) Colin, Traité de physiologie comparée des animaux, 3. éd., p. 821. Paris 1886.

2) v. Mering, Über die Funktion des Magens. Verhandl. des 12. Kongr. für innere Med. S. 471.

3) Moritz, Verhandl. des 12. Kongr. für innere Med. S. 487. 
übergeht, während die festen Massen im Magen zurückbleiben und hier d̀er weiteren Verdauung unterliegen."

Trotz dieser, auf Grund von Versuchsergebnissen ausgesprochenen Bemerkung von Moritz und späterer ähnlicher Untersuchungen desselben Autors ${ }^{1}$ ) ist eine klare Vorstellung über den Vorgang der Wasserentleerung bei gefültem Magen damals noch nicht gewonnen worden. Erst Cohnheim ${ }^{2}$ ) hat die Frage ihrer Lösung wesentlich näher gebracht, indem er seine bekannten Versuche am Duodenalfistelhund mit Fistel dicht hinter dem Pylorus ausführte und zeigte, dass auch bei vollem Magen das getrunkene Wasser rasch und in grossen Güssen entleert wird. Die wichtigste seiner Beobachtungen aber war, dass dieses entleerte Wasser fast vollkommen unvermischt, oft selbst ohne saure Reaktion angenommen zu haben, den Magen verliess. Von einer Mischung mit Mageninhalt, einer Ausschwemmung oder Durchspülung desselben konnte also nicht die Rede sein.

Unterdessen hatte man von den grundlegenden Versuchen von $\mathrm{Hirsch}$ und v. Mering ausgehend auch die wichtige Rolle der Pylorusreflexe für die Entleerung immer genauer erkannt und dabei auch die wichtige Tatsache ermittelt, dass Wasser sowohl vom Magen als auch vom Duodenum aus eine reflektorische Öfrnung des Sphincter pylori bewirkt. Damit war also auch der die rasche Entleerung ermöglichende Reflexmechanismus erkannt. Es blieb nur noch die Frage ungelöst, welchen Weg das Wasser durch den Magen nimmt, und welche $\mathrm{Be}$ wegungen der Magen hierbeì selbst ausführt.

Durch die Untersuchungen von $\mathrm{Kaufman} \mathrm{n}^{3}$ ) über Kontraktionsphänomene am Magen ist man neuerdings auf eine scheinbar einfache Lösung dieser Frage autmerksam gemacht worden. Schon Retzius ${ }^{4}$ ), Luschka ${ }^{5}$ ) und neuerdings Hasse und Strecker') haben aus dem Vorhandensein der schon längst bekannten sogenannten Kardiamuskelschleife oder Hufeisenschlinge des Magens auf die Möglichkeit der Bildung einer Rinne, die entlang der kleinen Kurvatur von der Kardia zum Pylorus führt, hingewiesen. Luschka, Kuss und Duval haben sogar den Übergang der Rinne in einen "mehr oder weniger" geschlossenen

1) Moritz, Studien über die motorische Tätigkeit des Magens. II. Zeitschr. für Biol. Bd. 42 S. 565.1901.

2) Cohnheim, Münchener med. Wochenschr. 1907 S. 2581.

3) R. Kaufmann, Anatomisch-experimentelle Studien über die Magenmuskulatur. Zeitschr. f. Heilk. Bd. 28 Heft 7. 1907. - R. Ka ufmann, Über Kontraktionsphänomene am Magen. Wiener klin. Wochenschr. Nr. 36. 1907.

4) A. Retzius, Bemerkungen über das Antrum pylori beim Menschen. Arch. f. Anat. u. Physiol. 1857 S. 74. - Retzius-Gyllenskoeld, Über die Fibrae obliquae des Magens. Arch. f. Anat. 1862.

5) Luschka, Anatomie des Menschen Bd. II S. 190. 1863.

6) Hasse und Strecker, Der menschliche Magen. Arch. f. Anat. (u. Physiol.) 1905 S. 33. - Hasse und Strecker, Der menschliche Magen. Anat. Anz. Bd. 25. 1904. - Strecker, Über den Verschl. der Cardia beim Menschen. Arch. f. Anat. (u. Physiol.) 1905. 
Kanal für wahrscheinlich erachtet ${ }^{1}$ ). Kaufmann gelang es an durch Einspritzung von Physostigmin in die V. jugularis in Kontraktionszustand versetzten Hundemägen eine solche Rinne nach Abpräparieren der Schleimhaut direkt darzustellen.

Was lag näher, als diese Rinne, deren Vorhandensein am durch Vergiftung kontrahierten Magen erwiesen war, als Weg der Flüssig. keiten und dünnflüssigen Massen anzusprechen, die, kaum in den Magen gelangt, auf diese Weise raseh und unvermischt zum Pylorus gelangen und diesen infolge der oben genannten Reflexwirkung ungehindert passieren konnten. In der Tat hat schon Retzius, wie ich weiter der Arbeit von Kaufmann entnehme, die Möglichkeit angedeutet, dass die Rinne zum Transport von Flüssigkeiten, dünnbreiigen Speisen usw. dienen könne. Nach Ka u fmann's ausführlichen Klarlegungen, durch die erst weitere Kreise mit diesen Verhältnissen bekannt wurden, hat man wohl ziemlich allgemein die Rinnenbildung zur Erklärung des $\mathrm{C}$ o h $\mathrm{n}$ h e i m'schen Versuches herangezogen ${ }^{2}$ ). Die Entleerung des Wassers ist danach so zu erklären, dass sich entlang der kleinen Kurvatur die Rinne oder ein geschlossener Kanal bildet, durch den das getrunkene Wasser, ohne sich mit dem Mageninhalt zu mischen, läuft.

Auch Ellenberger und ich haben diese Anschaung bereitwilligst angenommen und vertreten ${ }^{3}$ ); denn sie entsprach vorzüglich den vergleichend anatomisch und physiologisch bekannten und von uns vertretenen Anschauungen. In der Veterinäranatomie ist die Kardiamuskelschleife als wichtiger Bestandteil der Muskelschicht aller Haustiermägen ebenfalls längst bekannt $(G$ urlt beschreibt sie schon 1823 beim Pferde). Beim Pferde ist sie von allen Haustieren in mächtigster Weise entwickelt, und gerade dieses Tier nimmt sehr grosse Wassermassen auf, die eine Schädigung der Magenverdauung veranlassen würden, wenn sie nicht rasch entleert würden. Ausserdem ist es schon lange bekannt, dass von der aufgenommenen Nahrung beim Pferde stets ein kleiner Teil entlang der kleinen Kurvatur zum Pylorus zu gehen pflegt. Die Bildung der Rinne bzw. der Röhre wäre beim Pferde sehr leicht möglich, da die Kardiamuskelschleife sehr stark ist und ihre Schenkel weit bis zum Pylorus hinziehen

1) Literaturnachweise von Anm. 4-6 auf S. 413, zit. nach Kaufmann.

2) O. Cohnheim, Physiologie der Verdaung S. 18. Wiesbaden 1908.

3) Zuntz und Loewy's Lehrbuch der Physiologie S. 527. 
und an der Grenze zwischen Fundus- und Pylorusdrüsenzone in die Kreismuskulatur übergehen [Ellenberge $\left.\left.{ }^{1}\right)\right]$. Ausserdem steht aber der Magen des Pferdes mit seiner grossen Vormagenabteilung direkt an der Grenze zwischen einhöhligem und mehrhöhligem Magen. Bei den Tieren mit mehrböhligen Mägen ist eine solche Rinne direkt anatomisch vorgebildet vorhanden, die Speiserinne. Die Funktion dieser Rinne besteht im Transport dünnbreiiger und flüssiger Nahrungsbestandteile. Beim Hamster, der anch eine Speiserinne hat, konnte dies nachgewiesen werden ${ }^{2}$ ), bei den Wiederkäuern, deren Speiserinne den direkten Weg von der Kardia zum dritten Magen und im Verein mit der sich hier anschliessenden Rinne des dritten Magens (Psalterrinne) auch zum Labmagen darstellt, dient sie zum Transport der dünnbreiigen wiedergekauten Bissen, geringer Mengen der getrunkenen Flüssigkeiten und in den Fastenpausen zum Transport des abgeschluckten Speichels, wie man schon sehr lange annimmt. Wir beobachteten, dass sie, die gewöhnlich flach und offen ist, bei Reizung des peripheren Vagusstumpfes sich kontrahierte, ihre Lippen sich erhöhten und verdickten und so sich aus der Rinne ein geschlossenes Rohr formte. Da nun auch bei diesen Tieren eine, der Kardiamuskelschleife der einhöhligen Mägen vergleichbare Muskelpartie als Hauptmuskulatur der Rinne in erster Linie in Frage kommt, konnte von uns die Richtigkeit der Ka ufmann'schen Anschauung und Darlegung kaum bezweifelt werden.

Bei umfangreichen Versuchsreihen, die ich in Gemeinschaft mit Oberveterinär D r. Schattke über die Verdauung des normal gefütterten Truppenpferdes anstellte, fand sich, dass nach dem Tränken der Tiere der Mageninhalt noch stundenlang einen etwas höheren Wassergehalt als vor dem Tränken besass. Es musste also Tränkwassser im Magen zurückgeblieben sein. Dies veranlasste mich, die Frage zu prüfen, wo im Magen dieser Wasserrest und wo das aus dem Magen entleerte Wasser im Darm zu finden sei. Ich schritt daher zu Versuchen, bei denen den Tieren gefärbtes Wasser gereicht wurde. Diese Versuche hatten überraschend gute Ergebnisse. Das zur Färbung verwandte Malachitgrün störte die sonst so empfindlichen Pferde beim Trinken nicht und färbte sämtliche Inhaltsteile

1) Ell en berge r, Handb. der vergl. mikroskop. Anat. Bd. 3 S. 176. 1911.

2) A. Scheunert, Studien zur vergleichenden Verdauungsphysiologie. I. Pflüger's Arch. Bd. 121 S. 208. 1908; II. Bd. 139 S. 133.1911. 
von Magen und Darm, mit denen es in Berührung kam, so deutlich, dass man erstens stets sah, wo überhaupt Tränkwasser gewesen war, zweitens aber auch trotz des durch Heu bräunlich-grün gefärbten Darminhaltes ganz genau die Stelle des Darmes erkennen konnte, bis zu der das Wasser vorgedrungen war. Ich beobachtete nun, dass bei den getränkten Tieren stets die ganze Oberfläche des Mageninhaltes grün gefärbt und dass die grüne Farbe nur etwa $1-2 \mathrm{~cm}$ tief in die Inhaltsmasse eingedrungen war. Ferner zeigte sich stets eine durchgängige Grünfärbung des Inbaltes des Antrum pylori. Die Erwartung aber, an der kleinen Kurvatur entlang der hypothetischen Rinne besonders deutliche Grünfärbung des Inhaltes und der Schleimhaut zu finden, wurde getäuscht, im Gegenteil, die Umgebung der grossen Kurvatur war stets intensiver gefärbt als die der kleinen Kurvatur.

Diese Beobachtungen waren für mich die Veranlassung, genauere Versuche anzustellen.

\section{I. Über den Weg, den das Wasser im Magen genommen hat.}

(Mitbearbeitet von R. Otto, Assistent des Institutes.)

Die Methodik war nach dem Vorstehenden vorgezeichnet. Zur Darstellung des Wasserweges durch den Magen wurden die nach der Tötung der Tiere sorgfältig und sehr vorsichtig unter Vermeidung jeglichen Druckes exenterierten Mägen in Kältemischung gefroren, also derselbe Weg beschritten, den Gr ützner ${ }^{1}$ ) in seiner bekannten Arbeit zuerst gewiesen hat. Zur Feststellung der Länge der Darmabschnitte, die das getrunkene Wasser durcheilt hatte, wurde der Dünndarm in meterlangen Abschnitten unterbunden. Durch den gefüllten Magen wurden dann Längs- und Querschnitte angelegt, sowie nach Abziehen der Magenwand die Oberfläche des Mageninhaltes und die Magenschleimhaut betrachtet. Von den sich dabei ergebenden Bildern wurden getreue Pausen aufgenommen. Diese liegen den diesem Artikel beigegebenen Abbildungen zugrunde.

Die Versuche an Pferden beanspruchen deshalb besonderes Interesse, weil; wie schon eingangs erwähnt, das Pferd eine viel stärkere Kardiamuskelschleife wie z. B. Mensch und Hund besitzt, so dass die Rinnenbildung an der kleinen Kurvatur bei ihm besonders leicht zustande kommen müsste.

1) P. Grützner, Zum Mechanismus der Magenverdauung. Pflüger's Arch. Bd. 106 S. 463. 1905. 
Zur vorläufigen Prüfung der Richtigkeit der erwähnten gelegentJich gemachten Befunde wurde folgender orientierender Versuch ausgeführt.

Fin Pferd (Nr. 30) erhielt die militärisch übliche Morgenration von $1500 \mathrm{~g}$ Hafer und $200 \mathrm{~g}$ Häcksel. 60 Minuten nach Beginn der Mahlzeit und etwa 20-30 Minuten nach ihrer Beendigung wurde dem Pferde grüngefärbtes Wasser angeboten, wovon es 5 Liter trank. 20 Minuten nach dem letzten Schluck wurde es getötet, der Magen gefroren und in der Längsrichtung sowie quer durchsägt. Fig. 1 gibt das Bild ${ }^{1}$ ) des Längsschnittes. Danach hatte das Wasser den Mageninhalt umspült. Im Antrum pylori war die Färbung am stärksten und erstreckte sich auf dessen gesamten Inhalt, der total gefärbt war, in der Fundusregion und der kardiaseitigen Vormagenabteilung hingegen war die Farbe nur in die oberflächlichsten Portionen des Inhaltes eingedrungen, auch war besonders in dem linksseitigen Magenteil die Färbung nicht annähernd so intensiv, als in der Gegend des Antrum pylori. Vor allem war auffallend, dass an der Einmündungsstelle des Ösophagus und den benachbartenTeilen .der kleinen Kurvatur nur eine sehr unbedeutende Färbung zu sehen

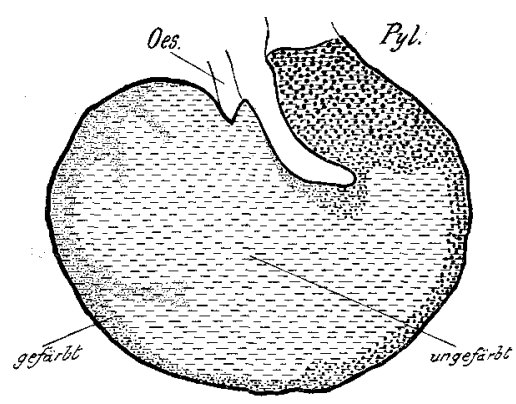

Fig. 1. war. Der Querschnitt durch die Magenmitte ergab ebenfalls nur eine Färbung der Randzone des Inhaltes. Anteile des Tränkwassers selbst waren im Magen nicht zu finden, hingegen war der Dünndarm bis zu drei Viertel seiner Länge mit grüngefärbtem wässerigem Inhait gefüllt. Da somit diese Versuche die gelegentlichen früheren Beobachtungen, nach denen das getrunkene Wasser den Mageninhalt in der Tat umspült hatte, bestätigten, gingen wir zu eingehenderen Versuchen über.

Ein 15 jähriger brauner Wallach (Nr. 31) erhielt $1500 \mathrm{~g}$ Hafer und $100 \mathrm{~g}$ Häcksel, die bis auf $300 \mathrm{~g}$ in 1 Stunde 10 Minuten verzehrt wurden. Dann erhielt das Tier $500 \mathrm{~g}$ Heu. Nachdem diese

1) Bei diesen ersten Versuchen (Pferd 30 und 33) legte ich noch keinen Wert auf die Anordnung des Mageninhaltes, sondern nur auf die Grünfärbung, deshalb sind die Schichtungsverhältnisse des Mageninhaltes nicht berücksichtigt. 
in 30 Minuten aufgenommen waren, trank das Tier 5 Liter malachitgrünes Wasser und wurde sofort danach getötet.

Fig. 2 gibt den Längsschnitt, Fig. 3 den Querschnitt des gefrorenen Magens wieder. Der Mageninhalt war von einer mehr oder weniger breiten, grüngefärbten Randzone umgeben. An der kleinen Kurvatur war der Saum schmal und blass, an der grossen Kurvatur war er breiter $(0,5-2 \mathrm{~cm})$, in der rechten Magenhälfte war die oberflächliche Inhaltsschicht stark gefärbt und in der Mitte der grossen Kurvatur scharf gegen den übrigen Mageninhalt abgesetzt. Nach dem Saccus caecus (der linksseitigen Vormagenabteilung) zu blasste die Färbung beträchtlich ab, ihre Abgrenzung war unscharf, und sie strahlte in einzelnen schwach gefärbten Zügen in den Inhalt aus. Der Querschnitt zeigte die stärkste Färbung an der grossen

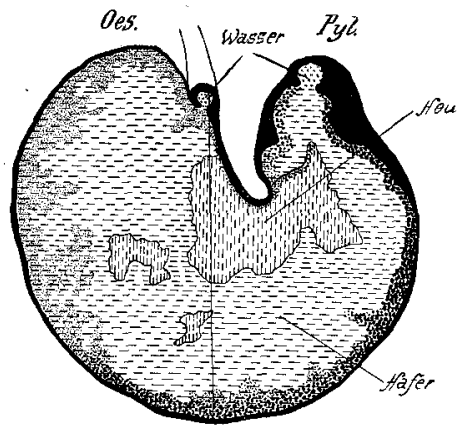

Fig. $2^{1}$ ).

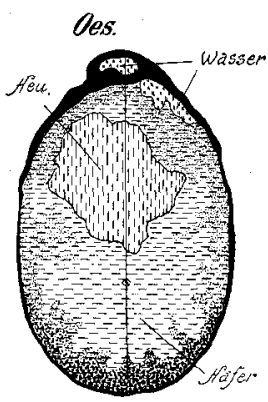

Fig. 3.

Kurvatur; an den Seitenflächeц war die Färbung schwächer. Die Oberfläche des Mageninhaltes zeigte sich nach Abziehen der Magenwand in toto gefärbt, doch war die Färbung links in der Vormagenabteilung bei weitem nicht so intensiv wie in der Magenmitte und im Pylorusteil. In bezug auf die Färbung der Schleimhaut ist zu. bemerken, dass die kutane Schleimhant der Vormagenabteilung die grüne Farbe nicht annahm; während die Drüsenschleimhaut sich färbte.

Bis zu einem Drittel der Länge des Dünndarmes war grüngefärbter Inhalt $\mathrm{zu}$ finden. Er betrug 1,75 Liter. Sofern eine Resorption nicht stattgefunden hatte, mussten also noch 3,25 Liter des

1) Bei sämtlichen Abbildungen sind die grüngefärbten Stellen durch Punktierung wiedergegeben. Die senkrechten durch die ganzen Zeichnungen gezogene Linien zeigen die Stelle des Quer- bzw. Längsschnittes an. 
Tränkwassers im Magen verblieben sein. Es besteht also der Einwand, dass, einen Transport des Wassers nach der Rinnenhypothese vorausgesetzt, das noch nicht entleerte Wasser am Antrum pylori oder sonstwo an der kleinen Kurvatur angesammelt gewesen wäre und bei dem im Gefolge der Tötung eintretenden Erschlaffen der Magenwände den Mageninhalt umflossen habe. Dieser Einwand wurde widerlegt durch einen analogen Versuch, bei dem die Tötung 40 Minuten nach dem letzten Schluck, also zu einer Zeit erfolgte, in der sicher alles Wasser dell Magen verlassen haben musste, soweit es nicht in den Inhalt eingedrungen war. Dieser in Magen zurückbleibende Teil ist darin natürlich nicht als freie Flüssigkeit, die zurückströmen könnte, vorhanden.

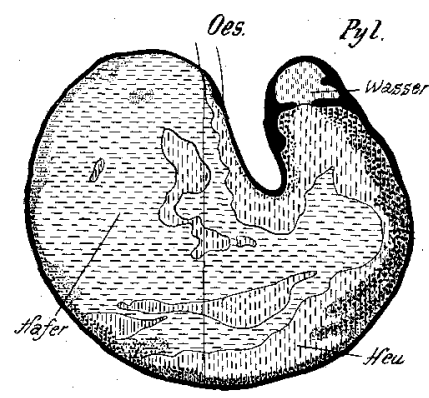

Fig. 4.

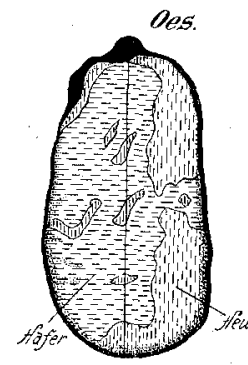

Fig. 5.

Pferd Nr. 32 (brauner Wallach) verzehrte $1500 \mathrm{~g}$ Hafer, $150 \mathrm{~g}$ Häcksel und $600 \mathrm{~g}$ Heu und trank dann 6,25 Liter grüngefärbtes Wasser. Wie die Untersuchung des Darminhaltes ergab, hatte das getrunkene Wasser den Magen grösstenteils verlassen. Der Inhalt und die Schleimhaut des Duodenums waren nämlich nur ganz schwach gefärbt. Erst nach $3,5 \mathrm{~m}$ begann die Färbung deutlicher zu werden, und nach weiteren $1,75 \mathrm{~m}$ war sie dunkelgrün geworden. In dem folgenden $5 \mathrm{~m}$ langen Darmstücke befand sich dunkelgrün gefärbter Inhalt. Die gefärbte Flüssigkeit war also schon weit im Darme vorgedrungen und im Moment der Tötung schon durch ein mehrere Meter langes Darmsttuck vom Magen getrennt.

Längs- (Fig. 4) und Querschnitt (Fig. 5) des Magens zeigten bezüglich der Färbung ein ganz ähnliches Bild wie beim vorher geschilderten Versuch. An der kleinen Kurvatur zeigt Fig. 4 die nur schwache und nicht scharf gegen den ungefärbten Inhalt abgesetzte Färbung. In der Pars pylorica an der grossen Kurvatur war die 
gefärbte Zone tiefgrün, ca. $3-5 \mathrm{~cm}$ breit und scharf begrenzt. Nach der Mitte der grossen Kurvatur zu wurde die Färbung schwächer, ihre Dicke geringer und die Abgrenzung undeutlich. In der Vormagenabteilung war die gefärbte Zone nur noch als feiner, $1-2 \mathrm{~mm}$. breiter Saum zn erkennen. Auch der Querschnitt zeigte die deutlichste Färbung an der grossen Kurvatur ( $3--5 \mathrm{~mm}$ dick), während an der kleinen Kurvatur die gefärbte Zone höchstens $1 \mathrm{~mm}$ breit: war. Die Oberfläche des Mageninbaltes war vollständig grün gefärbt.

Nach diesen beiden Versuchen kann also die Anschaung, dass die kleine Kurvatur die alleinige Strasse für das Tränkwasser sei, nicht gut aufrechterhalten werden. Immerhin bliebe noch folgende Möglichkeit. Die grossen Wassermassen bedürfen zu ihrer Entleerung einer gewissen Zeit und wahrscheinlich mehrerer einander folgender Öffnungen des Sphincter pylori. Man könnte nun annehmen, dass das Wasser zunächst gemäss der Rinnenhypothese zum Pylorus fliessen, dort sich ansammeln und bis zur Entleerung verbleiben würde. Diese Entleerung: geht, nach allem, was wir über die Mechanik des Magens wissen, mit peristaltischen Wellen Hand in Hand. Die Wellen sind im. Antrum stark und kräftig und könnten sehr wohl ein Zurückfluten. des noch nicht entleerten Wassers bewirken, wobei dieses möglicherweise den relativ festen Inbalt der links gelegenen Magenteile umspülen könnte, ohne tief in ihn einzudringen. Liefe der Vorgangin dieser Weise $a b$, so würde die Färbung der Oberfläche des Mageninhaltes erst ein sekundärer, durch die Peristaltik bewirkter Vorgang sein; den Weg der Flüssigkeit durch den Magen brauchte die Färbung: keineswegs anzugeben.

Es war also, um ein klares Bild über den Vorgang zu erhalten, nötig, den Weg der Flüssigkeit genauer zu verfolgen. Dies wurde dadurch zu erreichen gesucht, dass einige weitere Versuchstiere nur mit ganz geringen Wassermengen getränkt wurden. Gingen diese gemäss der Rinnenhypothese entlang der kleinen Kurvatur zum Pylorus, so war zu erwarten, dass sie ihrer geringen Menge wegen: rasch und vollständig entleert würden, ein Zurückstauen, wie oben geschildert, also nicht befürchtet zu werden brauchte.

Eine 16-18jährige Stute Nr. 33 bekam zunächst $250 \mathrm{~g}$ Kleie und $50 \mathrm{~g}$ Häcksel in angefeuchtetem Zustande und dann $750 \mathrm{~g}$ Heu. Hierauf wurde dem Tiere der gefüllte Tränkeimer gereicht, sofort aber nach wenigen Schlucken entfernt. Es hatte $1000 \mathrm{ccm}$ vom 
grün gefärbten Wasser getrunken. 1 Minute später erfolgte die Tötung.

Die Untersuchung des Dünndarmes ergab, dass die getrunkene Flüssigkeit schon entleert war, denn Darminhalt und Schleimhaut waren bis $2,5 \mathrm{~m}$ hinter dem Pylorus nur schwach gefärbt. Hierauf kam aber ein $1,80 \mathrm{~m}$ langes Stück mit tief grün gefärbtem Inhalt und grün gefärbter Schleimhaut, dann erfolgte $1,40 \mathrm{~m}$ lang Abnahme der Färbung.

Der Längsschnitt (Fig. 6) des gefrorenen Magens zeigte einen sehr schmalen grün gefärbten Saum, der den Inhalt in der rechten Magenhälfte umgab. Von der Ösophaguseinmündung zog nur ein schmaler feiner Strich entlang der kleinen Kurvatur zum Antrum pylori; an der Stelle der Umbiegung der Schleimhaut schob sich dieser in Form eines etwas breiteren und deutlich gefärbten Balkens

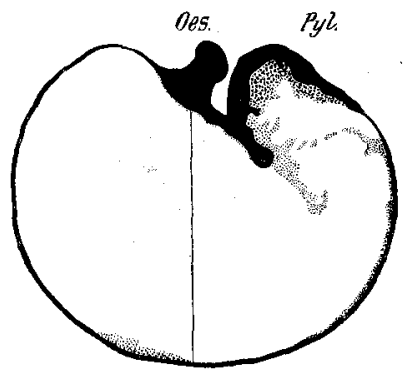

Fig. 6.

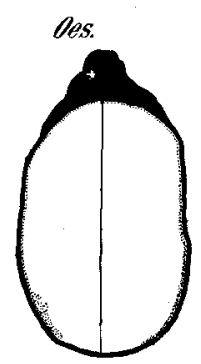

Fig. 7.

in den Inhalt hinein. An der Wand des Antrum war der Saum als unregelmässiger, etwas breiterer Streifen $\mathrm{zu}$ verfolgen. An der grossen Kurvatur war der Streifen kurz vor dem Pylorus auf eine 2-3 Finger breite Strecke unterbrochen, ebenso etwa $5 \mathrm{~cm}$ lang in der Gegend der Mitte der grossen Kurvatur. Fast an der ganzen linken Magenhälfte (Vormagenabteilung) fehlte hingegen die Grünfärbung. Der Querschnitt (Fig. 7) zeigte hiermit übereinstimmend einen feinen grünen Saum, der an der Seite der grossen Kurvatur etwa eine Handbreit unterbrochen war. Sehr instruktiv war die Betrachtung der Oberflächen (Fig. 8, 9). Der Inhalt der Vormagenabteilung zeigte fast durchgängig die natürliche Farbe des Futters, nur direkt links neben der Ösophagusmündung war eine ganz schwache Grünfärbung festzustellen. Die Oberfläcbe der pylorusseitigen zwei Drittel des Mageninhaltes war hingegen grün gefärbt, besonders stark die in der Verlängerung des Ösophagus nach der grossen Kurvatur 
zu liegenden Teile. In der Nähe der grossen Kurvatur war in der Magenmitte ein handtellergrosses Stück, ein ebensolches im Antrum pylori ungefärbt.

Aus der Betrachtung der Mageninhaltsoberfläche habe ich den Eindruck gewonnen, dass die Flüssigkeit nach ihrem Eintritt nach allen Seiten hin den Mageninhalt überflutet habe. Ein Teil ist in der ihm durch den Ösophagus vorgezeichneten Richtung nach der grossen Kurvatur hin über die beiden Seiten des Mageninhaltes geflossen, ein Teil ist sicher entlang der kleinen Kurvatur gegangen, obwohl dort die Oberfläche nur minimal gefärbt war. Offenbar haben, wie im zweiten Teil dieser Abhandlung auseinandergesetzt werden soll, am Ösophagus und der kleinen Kurvatur während des

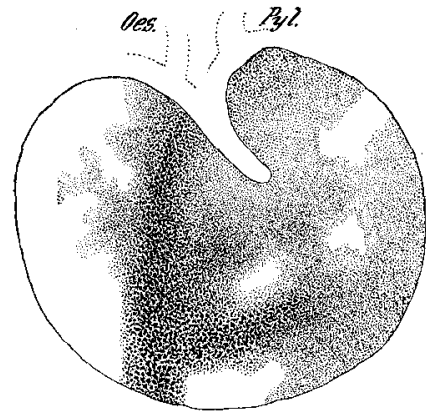

Fig. 8.

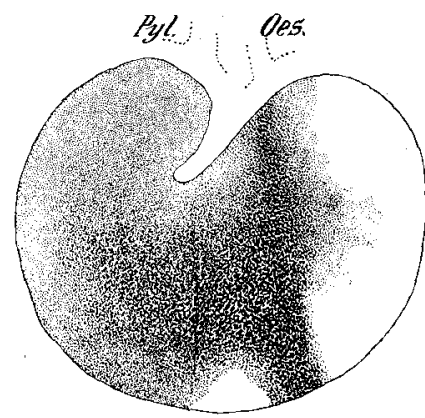

Fig. 9.

Tränkens andere Inhaltsteile, als sie der Längsschnitt zeigte, gelegen. Endlich ist auch vom Ösophagus nach dem blinden Ende hin ein weiterer kleinerer Teil der Flüssigkeit vorgedrungen.

Einen weiteren Versuch stellten wir zur Kontrolle an, bei dem das Versucbstier Nr. 34 nach der Versuchsmahlzeit von $1500 \mathrm{~g}$ Hafer, $150 \mathrm{~g}$ Häcksel und $750 \mathrm{~g}$ Heu $850 \mathrm{ccm}$ Wasser trank und 5 Minuten nach der Aufnahme getötet wurde. Da eine Entleerung von gefärbtem Wasser in den Darm noch nicht erfolgt war, fand sich vor dem Pylorus ejne grössere Menge sehr intensiv grün gefärbten Inhaltes.

Längs- (Fig. 10) und Querschnitt (Fig. 11) zeigten, dass das Tränkwasser nur an einigen Stellen bis zur grossen Kurvatur gelangt war. Ferner war hier im Mageninnern eine Verlagerung des Inhaltes derart eingetreten, dass die zuletzt genossene Heumenge, die auch während des Trinkens an der kleinen Kurvatur, wie ihre 
oberflächliche Grünfärbung bewies, gelegen hatte, durch Inhalt der Vormagenabteilung von der kleinen Kurvatur abgedrängt worden war, denn sie lag in der Mitte des Mageninhaltes. Ich werde hierauf später ausfübrlich zurückkommen. Die Betrachtung der Oberfläche des Mageninhaltes (Fig. 12) führte wieder zu der beim vorigen Versuche geäusserten Anschauung. Nach Eintritt in den Magen war das Wasser in der Richtung des Ösophagus über den Inhalt nach beiden Seiten hinweggelaufen, erreichte aber die grosse Kurvatur nur gegenüber dem Ösophagus. Andere Teile sind pyloruswärts an den Seiten des Magens und wohl auch an der kleinen Kurvatur, was infolge der erwähnten Verlagerung nicht genau verfolgt werden konnte, zum Pylorus vorgedrungen. Die hierher gelangte Wassermenge hatte sich mit dem Inhalte vermischt.

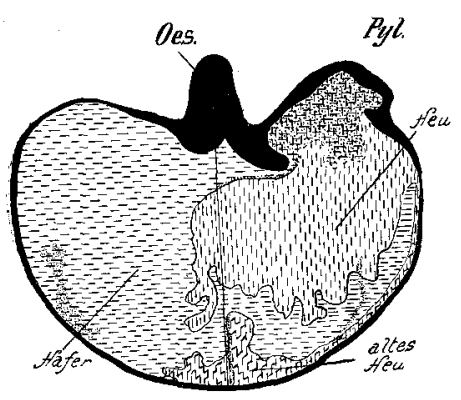

Fig. 10 .

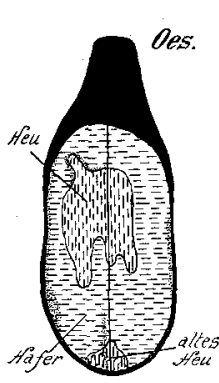

Fig. 11.

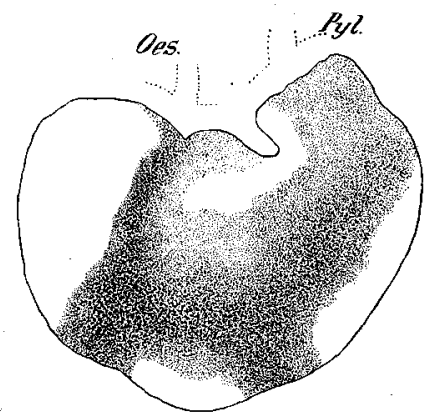

Fig. 12.

Eine $\mathrm{R} u \mathrm{ckstau} \mathrm{u} g$ derselben konnte ebenso wie heim vorigen auch bei diesem Versuche picht stattgefunden haben, da sonst die ungefärbten Stellen der Mageninhaltsoberflächen an der grossen Kurvatur und in der Nähe des Pylorus nicht hätten vorhanden sein können.

Bei den bisher geschilderten Versuchen erhielten die Tiere das Tränkwasser sofort nach beendeter Mahlzeit. Man könnte infolgedessen den (beim Pferde übrigens nicht ohne weiteres begründeten) Einwand gegen sie erheben, dass die Peristaltik in noch nicht genügender Weise eingesetzt habe und hierdurch sowie durch den grösseren Füllungsgrad des Magens eine Beeinflussung des Wassertransports stattgefunden habe. Wir haben aber diese Versuchsreihe mit Tränken bei starkgefülltem Magen absichtlich und unter anderen Gründen auch deshalb ausgeführt, weil nach Strecker (loc. cit.) die Bildung der hypothetischen Rinne durch Füllung des Magens und 
die dadurch bewirkte Spannung der Schenkel der Kardiamuskelschleife begünstigt wird.

Wir stellten, um der entgegengesetzten, von $\mathrm{K}$ a ufman $\mathrm{n}$ vertretenen Anschauung gerecht zu werden, eine weitere Versuchsreihe an, bei der das Tränken 30 Minuten nach Beendigung der Mahlzeit, also in den in voller Tätigkeit befindlichen Magen erfolgte.

Pf erd Nr. 35 erhielt zunächst $1000 \mathrm{~g}$ Heu, dann $1000 \mathrm{~g}$ Hafer + $100 \mathrm{~g}$ Häcksel und 30 Minuten nach der Mahlzeit $1250 \mathrm{ccm}$ grüngefärbtes Wasser. Die Tötung erfolgte 5 Minuten nach dem letzten Schluck. Wie die Betrachtung des Darminhaltes lehrte, war das Tränkwasser, soweit es überhaupt sofort entleert wird, schon in den.

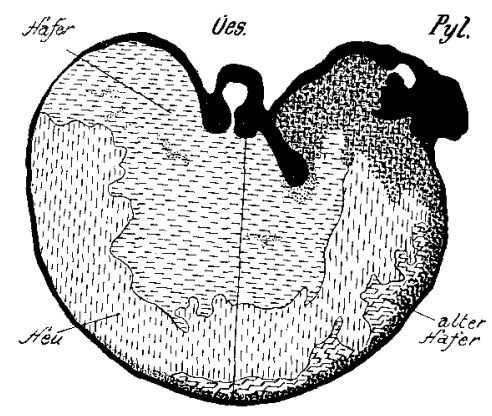

Fig. 13.

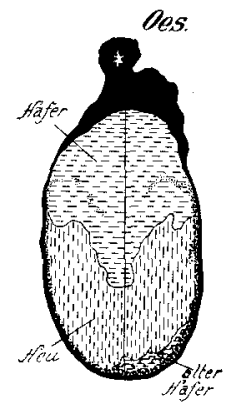

Fig. 14.

Darm eingetreten; denn das dem Magen zunächst liegende $1 \mathrm{~m}$ lange Darmstück hatte nur gering gefärbten Inhalt, erst die folgenden $3 \mathrm{~m}$ enthielten stark grün gefärbten Inhalt.

Im Antrum pylori war eine Durchmisehung und durchgängige Grünfärbung des Inhaltes zu beobachten, an der kleinen Kurvatur fehlte hingegen die Grünfärbung, wie Längs- (Fig. 13) und Querschnitt (Fig. 14) erkennen liessen. Die Ursache für das Fehlen der Grünfärbung an der kleinen Kurvatur ist in der schon mehrfach angedeuteten Verlagerung zu erblicken; wie auch auf den Abbildungen von Querschnitt und Längsschnitt zu erkennen ist, war im Innern der Hafermasse eine mit der kleinen Kurvatur gleichsinnig verlaufende grüne Zone festzustellen. An der grossen Kurvatur war ein schmaler, meist nur 1-2 mm dicker, tiefgefärbter grüner Saum an der Magenwandung zu beobachten. Er erstreckte sich auch noch in die Vormagenabteilung, aber nicht mehr weit hinein, das ganze linke Drittel des Magens war frei davon. 
Die Betrachtung der Oberfläche des Mageninhaltes liess erkennen, dass das Wasser, wie bej den vorherigen Versuchen, über die Inhaltsoberfläche nach der grossen Kurvatur und über die Seitenflächen pyloruswärts geflossen war. Das blinde Ende der Vormagenabteilung erwies sich frei von jeder Färbung.

Ein ähnlich angeordneter Versuch wurde zur Kontrolle ausgeführt. Pferd Nr. 36 erhielt $1500 \mathrm{~g}$ Hafer $+150 \mathrm{~g}$ Häcksel und $750 \mathrm{~g}$ Heu, 30 Minuten später $1250 \mathrm{ccm}$ malachitgrünes Wasser. Sofort nach dem letzten Schluck wurde es getötet. In den Darm war noch nicht viel von dem getrunkenen Wasser übergetreten, nur der in den ersten $90 \mathrm{~cm}$ enthaltene Inhalt war mässig grün gefärbt.

Der Längsschnitt (Fig. 15) zeigte, dass der Mageninhalt bis auf eine Strecke an der Einmündung des Ösophagus und an der kleinen

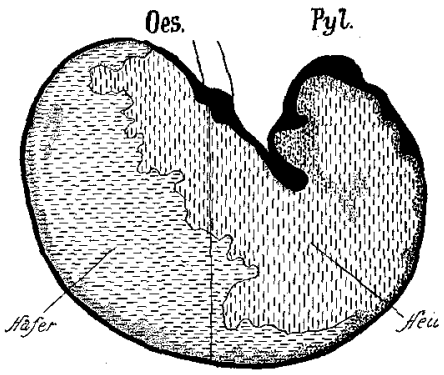

Fig. 15.

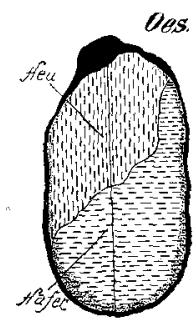

Fig. 16.

Kurvatur ganz von einer grünen Zone umgeben war. Am deutlichsten und scharf begrenzt war die Färbung dieser Zone im rechten Magenteil. Linksseitig war sie heller und die Begrenzung weniger scharf. An der Verlängerung der kleinen Kurvatur zog sich eine gefärbte Zone in den Inhalt hinein, die als Ausdruck der später zu erörternden Verlagerung anzusprechen ist. Der Querschnitt (Fig. 16) zeigte ein ganz entsprechendes Bild. Die Oberfläche des Mageninhalts wur überall grün gefärbt, die Färbung aber nicht gleichmässig, vielmehr war sie, von der Speiseröhre ausgehend, an den Seitenwänden des Magens nach der grossen Kurvatur und dem Pylorus zu am intensivsten. Ganz geringe Färbung zeigten die Oberfläche des Vormageninhaltes und die dicht am Pylorus gelegenen Inhaltsteile. Auch bei diesem Versuch scbeint das Wasser sich so über die Magenoberfläche ergossen zu haben, wie es nach den physikalischen Gesetzen obne Mitwirkung besonderer Mechanismen zu erwarten ist. 
Weiter stellten wir einen Versuch an, bei dem das in voller Verdauung befindliche Tier satt getränkt wurde.

P fer d Nr. 37 erhielt $2000 \mathrm{~g}$ Hafer und $200 \mathrm{~g}$ Häcksel, dann $750 \mathrm{~g}$ Heu. 30 Minuten nach der Mahlzeit wurde ihm zu trinken gereicht. Es nahm 12 Liter grüngefärbtes. Wasser auf und wurde 15 Minuten später getötet. Bis in den Blinddarm fand sich grüngefärbter Inhalt.

Auch bei diesem Versuch zeigte der Längsschnitt (Fig. 17) den grüngefärbten Saum, der den ganzen Mageninbalt mit Ausnahme einer kleinen Strecke an der kleinen Kurvatur umgab. Die Ursache hierfür liegt darin, dass durch einen Teil des erst genossenen Hafers das beim Tränken an der kleinen Kurvatur gelegene Heu von dieser und dem Ösophagus abgedrängt worden ist. Die am Rande des jetzt in der Mitte liegenden Heues befindliche gefärbte Zone zeigt

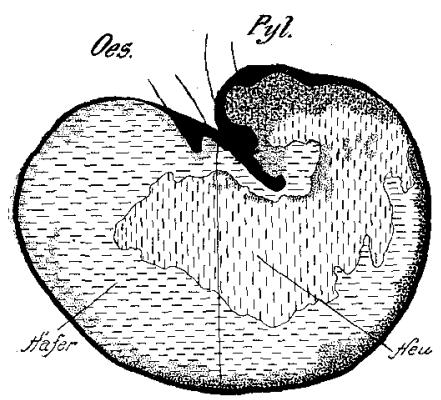

Fig. 17.

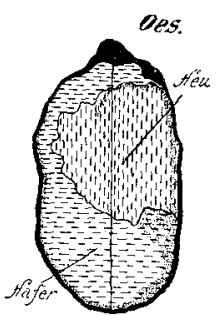

Fig. 18.

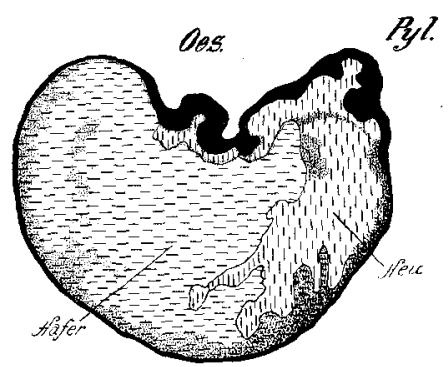

Fig. 19.

dies. Der Saum war in der Vormagenabteilung weniger stark gefärbt und unscharf, in der rechtsseitigen Magenabteilung scharf begrenzt und tiefgrün gefärbt. Die Oberfläche des Mageninhaltes war fast durchgängig gefärbt, eine Ausnahme machte nur das blinde Ende der Vormagenabteilung insofern, als es eine äusserst schwache Färbung aufwies und die Umgebung der kleinen Kurvatur, die ungुefärbt war. Das Wasser dürfte also auch bei diesem Versuch über den ganzen Mageninhait hinweggeflossen sein. Nur wenig hat den Inhalt der Vormagenabteilung umspült.

Endlich schoben wir bei einem Pferde (Nr. 38) die Tötung noch weiter hinaus. Dieses Tier erhielt $2500 \mathrm{~g}$ Hafer und $500 \mathrm{~g}$ Häcksel, dann $750 \mathrm{~g}$ Heu und 25 Minuten später 5 Liter grünes Wasser. Die Tötung erfolgte erst 2 Stunden nach dem Tränken. Auch bei diesem Versuche zeigte der Längsschnitt (Fig. 19), dass der Mageninhalt von einem grünen Saum umgeben war, der in der Vormagen- 
abteilung hell und unscharf war; die Oberfläche war total grün gefärbt. Der Versuch schloss sich also in seinen Ergebnissen vollkommen an die vorher genau geschilderten Versuche an.

Ich kann diesen Abschnitt nicht schliessen, ohne auf zwei Versuche hinzuweisen, die von den soeben geschilderten darin abweichen, dass bei ihnen der Mageninhalt ganz und gar gefärbt war.

Das eine Pferd (Nr. 29) hatte ca. 20 Minuten nach Beendigung einer aus $1500 \mathrm{~g}$ Hafer und $150 \mathrm{~g}$ Häcksel bestehenden Mahlzeit $9^{1 / 5}$ Liter Wasser getrunken und war sofort getötet worden. Das andere (Nr. 30) hatte nach einer Mahlzeit von $1500 \mathrm{~g}$ Hafer, $150 \mathrm{~g}$ Häcksel und $750 \mathrm{~g}$ Heu sofort $5^{1 / 2}$ Liter getrunken und war 1 Stunde später getötet worden. Die Ursache der Durchmischung dürfte beim ersten dieser beiden Pferde darin zu erblicken sein, dass die grosse aufgenommene Wassermenge im Moment der Tötung noch grösstenteils im Magen war.

Beim zweiten Versuche war der Magen trotz der reichlichen Mahlzeit nur mässig gefüllt, und es erscheint nicht unmöglich, dass die Durchmischung mit dem Trinkwasser (soweit es im Magen verblieben ist; bis ins Caecum fand sich grüngefärbter Inhalt) gerade infolge der geringen Füllung erfolgt ist. Hierfür sprechen vielleicht zwei weitere Versuche (Pferd 40 und 41), bei denen die Tiere vor der Mahlzeit getränkt wurden, ihre letzte Ration also ca. 12 bis 14 Stunden vor dem Tränken erhalten hatten. Pferd 40 trank 5 Liter, frass nach 5 Minuten $1500 \mathrm{~g}$ Hafer, $150 \mathrm{~g}$ Häcksel und $250 \mathrm{~g}$ Heu und wurde 10 Minuten darauf getötet. Pferd 41 trank $71 / 2$ Liter, frass nach 30 Minuten $1100 \mathrm{~g}$ Hafer und $750 \mathrm{~g}$ Heu und wurde nach 30 Minuten getötet. Bei diesen Tieren, deren Magen bei Beginn des Trinkens nur sehr wenig Inhalt hatte, war dieser Inhalt, soweit er neben dem neuen Versuchsfutter noch zugegen war, stets total grün gefärbt und nahm, an der grossen. Kurvatur liegend, einen Teil der pylorusseitigen Magenhälfte ein. Danach hatte sich also auch bei ziemlich leerem Magen das getrunkene Wasser teilweise mit dem Inhalt vermischt. Bei normal gefülltem Magen scheint aber eine solche Durchmischung nicht einzutreten. Jedenfalls haben wir auch bei den zu anderen Zwecken mit gefärbtem Wasser angestellten Tränkversuchen, die sich im ganzen auf weitere acht Tiere erstreckten, niemals eine totale Färbung beobachtet.

Durch diese Versuche dürfte der Beweis dafür erbracht sein, dass beim Pferde die Fortbewegung des getrunkenen Wassers derart. 
erfolgt, dass dieses zwischen Magenwand und Inhalt zum Pylorus fliesst und däbei den Mageninhalt umspült. Die Hauptmenge geht sicher nur durch den rechtsseitigen Magenteil, nur ein kleiner Teil hingegen in die teilweise höher als die Kardia gelegene Vormagenabteilung, deren Inhalt deshalb auch bei geringer Getränkmenge gar nicht oder nur teilweise vom Getränk berührt wird.

Es besteht für mich kein $\mathrm{Zweifel}$ darüber, dass auch entlang der kleinen Kurvatur ein Teil des 'Tränkwassers fliesst, also den kürzesten Weg zum Pylorus einschlägt. Darüber, wie gross dieser Anteil ist, lassen die Versuche natürlich keinen Schluss zu, überhaupt wird eine experimentelle Lösung dieser Frage höchst schwierig sein. In dieser Richtung irgendeine Behauptung aufzustellen, ist also, sofern man den Boden des Experimentes nicht verlassen will, nicht zulässig. Sicher ist nur, dass der Weg an der kleinen Kurvatur keinesfalls deralleinige Weg für Flüssigkeiten ist.

Ferner sprechen meine Versuche weder für noch gegen das Vorhandensein einer durch Kontraktion der Kardiamuskelschleife bei der Getränkaufnahme auftretenden Rinne. Sicher nicht vorhanden ist im Momente des Trinkens ein geschlossenes Rohr, welches die Flüssigkeit leitet; denn andernfalls könnte eine Umspülung des Mageninhaltes, wie ich sie gesehen habe, nicht stattfinden. Im übrigen bestreite ich weder das Vorhandensein der anatomischen Grundlagen einer Rinne noch die Möglichkeit ihrer Bildung unter bestimmten Verhältnissen.

Deshalb gerade möchte ich darauf hinweisen, dass eine Bildung und Mitwirkung der Rinne bei dem Getränketransport bisher überhaupt nicht bewiesen worden ist. Die Rinnenbildung, so bestechend sie ist, ist und bleibt eine Hypothese. Durch die Feststellung, dass die Rinne, auch wenn sie bestände, keinesfalls die alleinige Strasse des Tränkwassers darstellt, verliert die Rinnenfrage übrigens wesentlich an Bedeutung.

\section{II. Über im Gefolge der Wasseraufnahme auftretende Be- wegungen des Mageninhaltes.}

Bei der Schilderung der Versuche habe ich mich bisher fast lediglich auf ihre Deutung bezüglich des Wasserweges beschränkt, nicht aber die Schichtungsfrage des Futters erörtert. Auch in dieser Hinsicht bieten die Versuche höchst interessante Ergebnisse. Ehe 
auf diese eingegangen wird, müssen einige geschichtliche Daten angeführt werden.

Grützner ${ }^{1}$ ) hat aus seinen bekannten Versuchen über die Schichtung der Nahrung im Magen gefolgert, dass neue, zuletzt aufgenommene Nabrung in die Mitte der alten zu liegen komme, sich also nicht sofort mit der Schleimhaut des Magens berühre. Dieses soll erst dann der Fall sein, wenn die sie einhüllenden, von der Oberfläche nach innen verdauten Massen abgetragen und zum Pylorus und Darm befördert worden sind.

In einer späteren Arbeit habe ich dann dargetan, dass die Finschichtung der neuen in die alte Nahrung keineswegs allgemein und bei allen Tierarten die Regel ist, und auf die zahlreichen Faktoren, die bei dem Zustandekommen der Schichtung mitwirken können, hingewiesen. Besonders aber beim Pferde konnte ich, ebensowenig wie früher Ellenberger und andere eine solche konzentrische Schichtung finden. Vielmehr schichteten sich die Futtermittel so aufeinander, wie man sie reichte, und beruhrten, sofern ihre Menge gross genug war, auch stets sofort die Magenwand. Vor allem aber, und hierauf kommt es mir an dieser Stelle an, lag die zuletzt.gereichte Nahrung stets am Ösophaguseingange und der kleinen Kurvatur. Ich verweise dazu auf die in der zitierten Arbeit gegebenen Abbildungen. Auch bei späteren zahlreichen Versuchen über die Verdauung des Pferdes wurde immer wieder auf die Schichtungsverhältnisse im Magen geachtet und stets das gleiche gefunden. Um so mehr war ich erstaunt, als ich bei den vorliegenden Versuchen oftmals eine von den früher festgestellten Regeln abweichende Schichtung beobachtete und Bilder erhielt, nach denen in der Tat das zuletzt genossene Futter wenigstens zum Teil in der Mitte des alten lag, also so, wie es Grützner beschrieb. Damit aber nicht genug, bei einigen Versuchen war sogar die Schichtung zerstört und eine teilweise Vermischung des Inhaltes eingetreten. Zum Überblick sind die in Frage kommenden Längsschnitte hier nochmals den Zeiten entsprechend, die zwischen Beendigung des Trinkens und der Tötung verflossen sind, nebeneinander gestellt. Sie zeigen sich so, wenn man sie gemeinsam betrachtet,

1) P. Grützner, Zum Mechanismus der Magenverdauung. Pflüger's Arch. Bd. 106 S. 463.1905.

2) Arthur Sche un ert, Zum Mechanismus der Magenverdaung. Pflüger's Arch. Bd. 114 S. 90. 1906. 


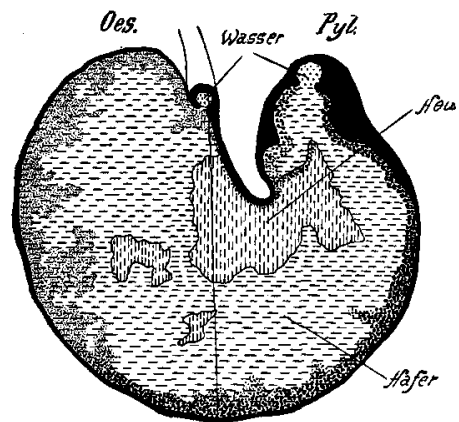

Fig. 20.

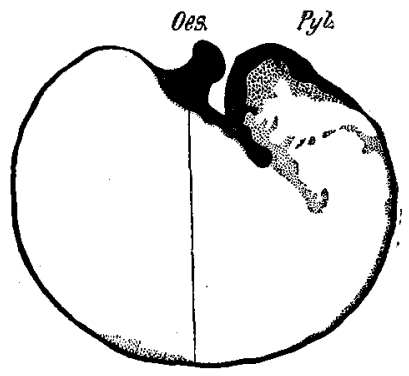

Fig. 22 .

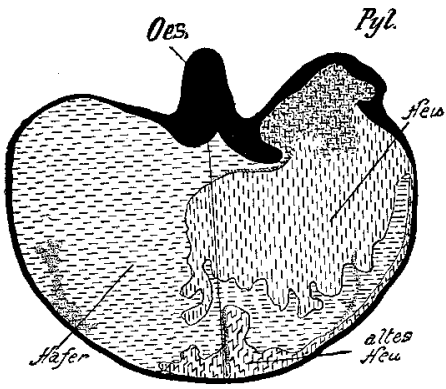

Fig. 24.

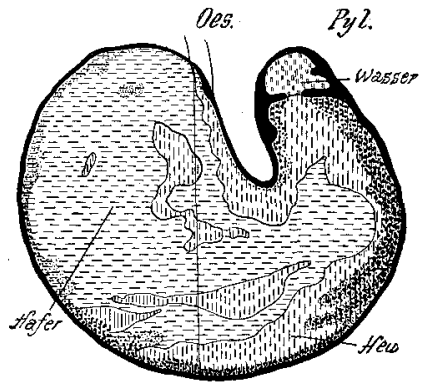

Fig. 26.

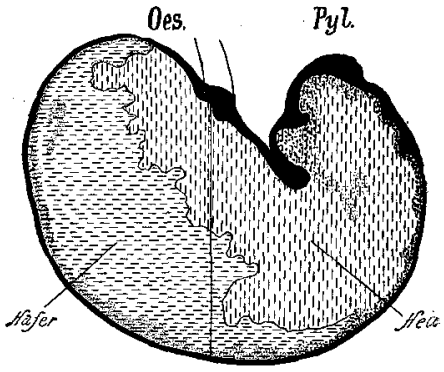

Fig. 21.

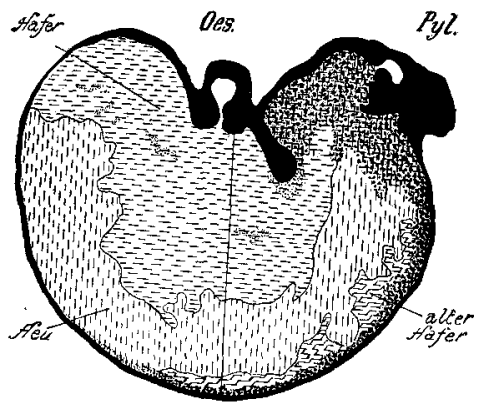

Fig. 23.

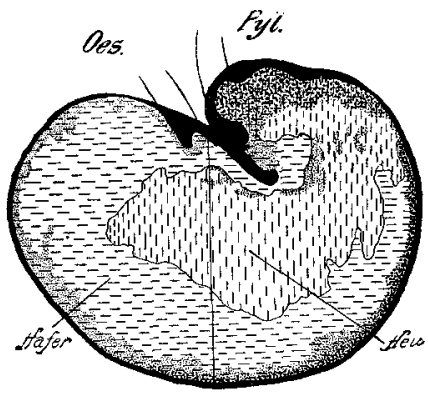

Fig. 25.

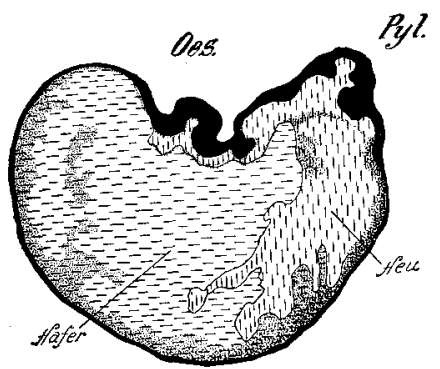

Fig. 27. 
als Phasen eines Bewegungsvorganges, denen die Futtermassen unterworfen gewesen sind.

Die erste Abbildung zeigt uns den Mageninhalt eines direkt nach dem Fressen getränkten ( 5 Liter) und sofort getöteten Pferdes, Das zuletzt genossene Heu liegt noch nahe der Einmündung des Ösophagus an der kleinen Kurvatur. Es ist aber schon eine Verlagerung desselben eingetreten, und zwar ist es von seinem ursprünglichen Liegeplatz nach rechts pyloruswärts verschoben worden. Dabei sind andere Inhaltsteile, die während des Tränkens nicht an der Ösophagusmündung und am Anfang der kleinen Kurvatur gelegen haben, dorthin geschoben worden. Wie wäre es sonst möglich, dass an der Kardia und direkt daneben an der kleinen Kurvatur nur eine ganz minimale, kaum sichtbare Grünfärbung featzustellen war? Weiter aber ist die Heumasse bei dem Schub nach rechts in den Inhalt des Antrum pylori hineingepresst worden. Das beweist der deutlich grüne, etwa $1 / 2 \mathrm{~cm}$ breite Saum, den diese Heumasse besitzt. Der Druck, den diese Verlagerung bewirkt hat, hat gleichzeitig ein Stück der Heumasse im linken Magendrittel abgesprengt.

Das nächste Bild (Fig. 21) zeigt dieselben Verlagerungen des Inhaltes, obwohl hier das Trinken $1 / 2$ Stunde nach der Mahlzeit erfolgte und nur wenig getrunken wurde. Gerade am Ösophagus und um ihn herum ist keine Grünfärbung zu sehen, dorthin müssen also von innen heraus, nachdem das Wasser abgelaufen war, ungefärbte Inhaltsteile geschoben worden sein. Die Druckrichtung von links nach rechts erkennt man aus der Art der Verlagerung der kompakten mit grünem Saum versehenen Heumasse. Diese Färbung kann meines Erachtens nach nur so erklärt werden, dass die gefärbten Teile während des Tränkens der kleinen Kurvatur anlagen, deshalb gefärbt wurden und nachher verlagert worden sind.

Der nächste Magen (Pferd 33, sofort nach dem Fressen getränkt und 1 Minute nach dem Trinken getötet) zeigt dasselbe Bild (Fig. 22). Das Fehlen jeder stärkeren Färbung in der Nähe der Kardia, das Vorhandensein eines gefärbten Streifens in der Verlängerung der kleinen Kurvatur deuten auf den gleichen Bewegungsvorgang hin.

5 Minuten nach dem Trinken wurde Pferd 35 getötet, dessen Magenlängsschnitt die folgende Abbildung $\mathrm{Nr} .23$ wiedergibt. Obwohl dieses Tier zuerst $\mathrm{Heu}$ und dann $\mathrm{Hafer}$ erhielt, also umgekehrt wie die anderen Tiere gefüttert wurde, finden wir, wenn auch nicht so deutlich, dasselbe Bild. An der Einmündung des Ösophagus und an 
der kleinen Kurvatur ist kaum Färbung zu sehen, wohl aber finden sich im Innern der Hafermasse, einige Zentimeter unter der Magenwand, einige gefärbte Stellen, die zu der Haferschicht gehören, die während des Tränkens an der kleinen Kurvatur anlag. Auf dem Bilde des Querschnittes (vgl. Fig. 14) sieht man dies noch deutlicher. Da der Hafer im Magen lange nicht eine so kompakte Masse wie das Heu bildet und infolge der Fütterung in diesem Falle der zuletzt gefütterte Hafer durch von links und unten kommenden Hafer abgedrängt worden ist, ist die gefärbte Zone nicht so deutlich. Andererseits zeigt der Versuch, dass der Bewegungsvorgang mit der Futterfolge, wie man etwa meinen könnte, nichts zu tun hat.

Bei dem ebenfalls 5 Minuten nach dem Tränken getöteten Pferde 34 befindet sich die Verlagerung in einem etwas fortgeschritteneren Stadium (Fig. 24). Das ursprünglich während des Trinkens am Ösophagus und der kleinen Kurvatur gelagerte Heu ist nach rechts verschoben und von der kleinen Kurvatur durch von links und unten her vorgeschobene Hafermassen abgedrängt worden. Dass es beim Tränken an der kleinen Kurvatur gelegen hat, beweist der erhalten gebliebene gefärbte Saum; der Beweis dafür, dass die Abtrennung erst nach dem Trinken erfolgt ist, ist darin zu erblicken, dass der nunmehr an der Ösophagusmündung liegende Hafer ungefärbt ist.

Fin weiteres Stadium des Bewegungsvorganges gibt das Längsschnittbild des Magens von Pferd 37, welches 15 Minuten nach dem Tränken getötet war. Es unterscheidet sich von Fig. 24 nur dadurch, dass die Abdrängung des zuletzt gegebenen Heues noch weiter fortgeschritten ist; der zwischen Heu und kleine Kurvatur getretene Hafer bildet eine zusammenhängende Zone, die bis ins Antrum pylori reicht.

Die zwei folgenden Längsschnitte endlich geben in übereinstimmender Weise die Lagerung wieder, die der Mageninhalt nach Ablauf der oben geschilderten Umwälzung annimmt.

Bei Pferd 32 (Fig. 26) erfolgte die Tötung 40 Minuten, bei Pferd 38 (Fig. 27) endlich 2 Stunden nach dem letzten Schluck. Trotz dieser zeitlichen Differenz stimmen die Bilder im grossen und ganzen untereinander und mit den oben beschriebenen so gut überein, dass ich davon äusserst überrascht war, da die betreffenden Versuche zeitlich weit auseinander lagen und ursprünglich zu anderen Zwecken angestellt wurden, Das zuletzt gefressene Heu, welches während 
des Tränkens in der Magenmitte lag, ist so verlagert worden, dass es jetzt nahe der grossen Kurvatur des rechten Magendrittels liegt. Die Abdrängung ist durch Vordringen des durch Druck von links und unten in Bewegung gesetzten Inhaltes des linken Magendrittels erfolgt. Wie ausserordentlich diese Umwälzung gewesen ist, zeigen die Fig. Nr. 24, 26 und 27, aus denen hervorgeht, dass sogar eine teilweise Vermischung von Hafer und Heu eingetreten ist. Bei Magen Nr. 26 und Nr. 27 ist ein kleiner Teil des Heues an seiner ursprünglichen Lagerstelle oder nahe derselben liegen geblieben, und der von links vorgetriebene aus Hafer bestehende Inhalt hat sich unter dem Heu hinweg und in dasselbe hineingeschoben.

Aus diesen Versuchen ist $\mathrm{zu}$ schliessen, dass im Gefolge der Getränkaufnahme ein mit mächtiger Kraftentwicklung einhergehender Bewegungsvorgang der Magenwandung abläuft, der eine eigenartige Verlagerung des Inhaltes, sogar unter teilweiser Vermischung desselben, verursacht. Zeitlich tritt dieser Vorgang erst nach Beendigung des Trinkens ein, denn die beim Töten an der Einmündung des Ösophagus lagernden Inhaltsteile waren meist gar nicht oder nur äusserst schwach gefärbt. Das Trinken selbst muss also der Reiz sein, der den Vorgang auslöst, denn nur im Gefolge des Trinkens, sonst niemals, ist die geschilderte Verlagerung zu beobachten; auch ist es gleichgültig, ob das betreffende Tier sofort nach dem Fressen oder später getränkt wurde.

Wir müssen daraus auf eine besondere Tätigkeit der Muskulatur der linken Magenseite, also der Vormagenabteilung des Pferdes schliessen. In der Tat haben Ellenberger und Seber ${ }^{1}$ nachgewiesen, dass sich die Muskulatur der Vormagenabteilung des Pferdes besonders an der am meisten vorgewölbten Partie durch besondere Dicke auszeichnet. Ausserdem stellten sie eine in der Wand der Vormagenabteilung an deren Grenze zum Drüsenmagenteil verlaufende Muskelverdickung fest, die bandartig den ganzen Magen umfasst und bei ihrer Kontraktion eine Abschnürung der Vormagenabteilung vom Drüsenmagen bewirken könnte, und die sie deshalb Sphincter ventriculi (Sphincter der eigentlichen Drüsenmagenabteilung) nannten. Beim Zusammenwirken dieses Sphincter ventriculi und der kräftigen Vormagenmuskulatur dürfte der Vormageninhalt unter einen Druck gesetzt werden, der dem aus den geschilderten

1) Ellen berger, Handb. der vergl. mikroskop. Anat. Bd. 3 S. 177. 1911. 
Versuchen Gefolgerten entspricht. Auch der grosse Reichtum der Vormagenabteilung an elastischem Gewebe dürfte hierbei als unterstützendes Moment in Frage kommen.

Es ist vielleicht nicht überflüssig, zu betonen, dass von einer Erschlaffung der Vormagenabteilung während des Triukens zur Aufnahme des Getränkes nicht die Rede sein kann, da der Inhalt der Vormagenabteilung mit dem Getränk manchmal gar nicht in Beruhrung kommt.

\section{Zusammenfassung.}

Die wichtigsten durch die vorliegenden Versuche gewonnenen Resultate über den Magenmechanismus des Pferdes bei der Getränkaufnahme möchte ich in folgenden Sätzen erblicken.

1. Die in den Magen des Pferdes beim Trinken unter gewöhnlichen Verhältnissen aufgenommene Wassermenge wird, soweit sie rasch entleert wird, derart durch den Magen hindurch und aus ihm herausbefördert, dass sie, zwischen Inhalt und Magenwand hindurehfliessend, den ganzen Mageninhalt umspült. Die Hauptmenge fliesst dabei in der Eintrittsrichtung des Ösophagus an der kleinen Kurvatur, an den Seitenflächen und über diese zur grossen Kurvatur und an dieser entlang. Durch die links von der Ösophagusmündung gelegene Vormagenabteilung nimmt nur ein geringerer Teil des Getränkes und nur bei Aufnahme grösserer Quantitäten seinen Weg.

2. Inwieweit die kleine Kurvatur als $\mathrm{Hauptweg}$ der Flüssigkeit angesehen werden kann, lässt sich nach den Versuchen ebensowenig beurteilen wie die Berechtigung der Annahme, dass im Gefolge der Getränkaufnahme an der kleinen Kurvatur eine Rinne durch die Kontraktion der Kardiamuskelschleife (Hufeisenschlinge) entsteht.

Hält man daran fest, dass vorläufig beides Hypothesen sind, so ist im Hinblick auf die zahlreichen Wahrscheinlichkeitsgründe nicht einzusehen, warum man vorläufig nicht an der Annahme einer vorzugsweisen Benutzung der kleinen Kurvatur als Weg bei der Getränkentleerung festhalten sollte.

Jedenfalls beweisen die Versuche aber, dass die kleine Kurvatur nicht der alleinige Weg des Getränkes ist, dass also ein einfaches Vorbeifliessen des getrunkenen und sofort zur Entleerung kommenden Wassers am Inhalt entlang der kleinen Kurvatur nicht stattfindet. Sonst könnte der Inhalt nicht so vollständig umspült werden, wie es bei den Versuchstieren der Fall war. 
Ứber den Magenmechanismus des Pferdes bei der Getränkaufnahme. 435

Aus gleichen Gründen erachte ich die Versuche als beweisend dafür, dass die Fortleitung des Wassers durch einen geschlossenen Kanal; der etwa durch Umbildung der Rinne entstehen könnte, keinesfalls erfolgt. Ob die Bildung einer of fenen Rinne erfolgt oder nicht, bleibt dahingestellt.

3. Im Gefolge der Getränkaufnahme tritt ein sich auf die erste Zeit nach dem letzten Schluck erstreckender Bewegungsvorgang des Magens und des Mageninhaltes ein. Er besteht darin, dass durch Druck, ausgehend von der Muskulatur der Vormagenabteilung und dem Sphincter ventriculi, der im Innern derselben lagernde Inhalt entlang der kleinen Kurvatur nach rechts pyloruswärts gedrängt wird, so dass die während des Trinkens dort liegenden Inhaltsteile nach rechts und innen verlagert werden.

\section{Nachschrift.}

Über analoge Versuche an Hunden beabsichtige ich in kürzester Zeit $\mathrm{zu}$ berichten. 